\title{
Floral and insect-induced volatile formation in Arabidopsis lyrata ssp. petraea, a perennial, outcrossing relative of $A$. thaliana
}

\author{
Christian Abel • Maria Clauss • Andrea Schaub • \\ Jonathan Gershenzon • Dorothea Tholl
}

Received: 27 October 2008 / Accepted: 5 March 2009 / Published online: 26 March 2009

(C) The Author(s) 2009. This article is published with open access at Springerlink.com

\begin{abstract}
Volatile organic compounds have been reported to serve some important roles in plant communication with other organisms, but little is known about the biological functions of most of these substances. To gain insight into this problem, we have compared differences in floral and vegetative volatiles between two closely related plant species with different life histories. The self-pollinating annual, Arabidopsis thaliana, and its relative, the outcrossing perennial, Arabidopsis lyrata, have markedly divergent life cycles and breeding systems. We show that these differences are in part reflected in the formation of distinct volatile mixtures in flowers and foliage. Volatiles emitted from flowers of a German A. lyrata ssp. petraea population are dominated by benzenoid compounds in contrast to the previously described sesquiterpene-dominated emissions of A. thaliana flowers. Flowers of A. lyrata ssp. petraea release benzenoid volatiles in a diurnal rhythm with highest emission rates at midday coinciding with observed visitations of pollinating insects. Insect feeding on leaves of A. lyrata ssp. petraea causes a variable release of the volatiles methyl salicylate, $\mathrm{C}_{11^{-}}$and $\mathrm{C}_{16}$-homoterpenes, nerolidol, plus the sesquiterpene (E)- $\beta$-caryophyllene, which in
\end{abstract}

C. Abel $\cdot$ M. Clauss $\cdot$ J. Gershenzon $\cdot$ D. Tholl

Max Planck Institute for Chemical Ecology,

Hans-Knoell Str. 8, 07745 Jena, Germany

A. Schaub

Ionicon Analytik GmbH, Technikerstr. 21 A,

6020 Innsbruck, Austria

Present Address:

D. Tholl $(\varangle)$

Virginia Polytechnic Institute and State University,

408 Latham Hall, AgQuad Lane, Blacksburg, VA 24061, USA

e-mail: tholl@vt.edu
A. thaliana is emitted exclusively from flowers. An insectinduced gene (AlCarS) with high sequence similarity to the florally expressed (E)- $\beta$-caryophyllene synthase (AtTPS 21 ) from A. thaliana was identified from individuals of a German A. lyrata ssp. petraea population. Recombinant AlCarS converts the sesquiterpene precursor, farnesyl diphosphate, into $(E)$ - $\beta$-caryophyllene with $\alpha$-humulene and $\alpha$-copaene as minor products indicating its close functional relationship to the A. thaliana AtTPS21. Differential regulation of these genes in flowers and foliage is consistent with the different functions of volatiles in the two Arabidopsis species.

Keywords Arabidopsis . Diurnal volatile emission . Herbivory $\cdot$ Terpenoid $\cdot$ Terpene synthase $\cdot$ Volatiles
Abbreviations
AlCarS Arabidopsis lyrata ssp. petraea
(E)- $\beta$-caryophyllene synthase
AtTPS21 A. thaliana terpene synthase 21 (At5g23960)
DMNT 4,8-Dimethyl-1,3,7-nonatriene
FPP Farnesyl diphosphate
GC-MS Gas chromatography-mass spectrometry
PTR-MS Proton-transfer-reaction mass spectrometry
RT-PCR Reverse transcriptase polymerase chain reaction
SPME Solid-phase micro extraction
TMTT 4,7,8-Trimethyl-1,3,7,11-tridecatetraene

\section{Introduction}

Plants release a large variety of volatile organic compounds into the environment as part of their interaction with other organisms. By providing information across distances, 
plant volatiles are implicated in multiple functions such as the attraction of pollinators or defense against herbivorous insects via direct deterrence or attraction of insect enemies (Kessler and Baldwin 2001; Pichersky and Gershenzon 2002; Dicke et al. 2003; Rasmann et al. 2005). Floral and vegetative volatile blends and their patterns of emission can exhibit significant variation among or within plant species (Raguso et al. 2003; Hoballah et al. 2005; Knudsen et al. 2006; Roeder et al. 2007). These phenotypic differences are likely to be the result of natural selection and adaptation to different pollinators, herbivores and herbivore enemies. Yet, only limited biochemical and genetic information is available regarding the evolution of volatile chemical traits and their intra- and inter-specific variation. Studies on the molecular mechanisms underlying the divergence of volatile profiles have focused primarily on cultivated plants. For example, relative differences in the content of volatile monoterpenes and sesquiterpenes in different basil varieties are correlated with transcript levels of distinct terpene synthase genes (Iijima et al. 2004). Another comprehensive analysis of insect-induced volatiles performed on several maize inbred lines revealed substantial genetic variability in vegetative odor emissions (Gouinguene et al. 2001; Degen et al. 2004).

Few molecular studies have been undertaken to compare floral and vegetative volatile emissions among related wild plant species. To gain further insight into volatile trait differences of such species, we have chosen Arabidopsis thaliana and its close relative Arabidopsis lyrata for study. The two species diverged approximately 5 million years ago from a common ancestor into lineages with distinctively different life forms and breeding systems (Koch et al. 2000; Clauss and Koch 2006). While A. thaliana is a predominantly self-pollinating, weedy annual, A. lyrata is a strict outcrossing perennial (Nasrallah 2000). Gene sequence comparisons between the two species indicate an average species divergence at synonymous and non-synonymous sites of approximately 15 and $1 \%$, respectively (Clauss and Koch 2006). Such high levels of nucleotide identity should facilitate direct comparisons of the structure and regulation of orthologous genes involved in volatile formation.

The floral and vegetative volatiles of A. thaliana have been extensively analyzed. The small $(0.4 \mathrm{~cm})$ white flowers of this species emit low amounts of monoterpenes and sesquiterpenes with $(E)$ - $\beta$-caryophyllene as the predominant compound (Chen et al. 2003; Tholl et al. 2005). Rosette leaves of the $A$. thaliana ecotype Columbia release a simple blend of the sesquiterpene $\alpha$-farnesene, the $\mathrm{C}_{16}$ homoterpene 4,7,8-trimethyl-1,3,7,11-tridecatetraene (TMTT) and the benzenoid compound methyl salicylate in response to attack by insect herbivores and in response to fungal elicitors (Van Poecke et al. 2001; Herde et al. 2008). Most genes and enzymes responsible for the formation of floral and leaf volatiles of $A$. thaliana have now been identified (Chen et al. 2003; Fäldt et al. 2003; Tholl et al. 2005) allowing comparisons to orthologues in the A. lyrata genome.

Much less is known about the volatiles of the flowers and foliage of A. lyrata and their biosynthesis. The white flowers of this species are approximately $1 \mathrm{~cm}$ in diameter (Clauss and Koch 2006). The floral scent of the North American subspecies, A. lyrata ssp. lyrata, has been reported to consist primarily of benzenoid compounds (Peer and Murphy 2003). However, volatiles of the European subspecies, A. lyrata ssp. petraea, have not been determined. Based on the perennial life-form and outcrossing nature of $A$. lyrata, we expected that the floral and vegetative volatile blends emitted by $A$. lyrata ssp. petraea would differ from those of $A$. thaliana. Given the genetic diversity within A. lyrata ssp. petraea populations (Clauss and Mitchell-Olds 2006), we also predicted to find intrapopulation variability of volatiles. Here, we present evidence for qualitative and quantitative differences in floral and insect-induced volatile traits within two German populations of A. lyrata ssp. petraea. We demonstrate that volatiles emitted from A. lyrata ssp. petraea flowers are vastly different from floral volatiles of $A$. thaliana and are emitted in a diurnal rhythm. Furthermore, we show that, in contrast to previous analyses from $A$. thaliana, herbivory on A. lyrata ssp. petraea rosette leaves induces the emission of (E)- $\beta$-caryophyllene as a variable trait and that a presumed orthologue of the floral-specific $A$. thaliana $(E)$ - $\beta$-caryophyllene synthase (AtTPS21) is responsible for the production of $(E)$ - $\beta$-caryophyllene in A. lyrata ssp. petraea leaves. The ecological relevance of the observed differences in volatile formation between the two species is discussed with respect to their life forms and breeding systems. The results pave the way for detailed genetic comparison of the floral and vegetative volatile phenotypes of the two Arabidopsis species.

\section{Materials and methods}

Plant material and growth conditions

Seeds from Arabidopsis lyrata ssp. petraea (L.) Hiitonen were originally collected from plants of two German populations close to Plech (northern Bavaria, Germany) and Neutras (northern Bavaria, Germany). Sampling of seeds was done from randomly chosen plants, each separated by more than $1 \mathrm{~m}$ from each other to avoid sampling from clonal relatives (Clauss and Mitchell-Olds 2006). Seeds were germinated as described (Clauss and Mitchell-Olds 2006). Plants were cultivated in plant climate rooms at 
$22^{\circ} \mathrm{C}, 55 \%$ relative humidity, and $160 \mu \mathrm{mol} \mathrm{m}{ }^{-2} \mathrm{~s}^{-1}$ photosynthetically active radiation (PAR) in a $10 \mathrm{~h} \mathrm{light/14 \textrm {h }}$ dark cycle. Individual plants of the Plech population used in this study were derived from controlled crosses of laboratory-grown sire and dam plants (Clauss et al. 2006) and propagated vegetatively by separating shoots that emerged from roots. Individuals from both populations were used for experiments after at least 3 months of growth.

\section{Insect feeding experiments}

Herbivory experiments were performed with larvae of a Plutella xylostella G88 colony. Larvae were reared on artificial diet with a wheat germ base at $27^{\circ} \mathrm{C}$ under a $18 \mathrm{~h}$ light/6 h dark cycle (Ratzka et al. 2002). Feeding experiments were performed in a dynamic headspace volatile collection system (see "Volatile collection and analysis" below). On day 0 , potted plants were transferred into the glass chambers of the volatile collection system. On the morning of day 1, 20-30 P. xylostella larvae in the third or fourth instar were placed on individual plants and allowed to feed for $24 \mathrm{~h}$. Volatiles were collected in the light for $8 \mathrm{~h}$ from the start of insect feeding and, after removal of the larvae, for the same time period on day 2 .

Volatile collection and analysis

A dynamic headspace volatile collection system was used for sampling volatiles from insect-damaged plants. The system consisting of six 3-L glass containers was operated in a plant climate chamber at $22^{\circ} \mathrm{C}, 70 \%$ relative humidity, and $150 \mu \mathrm{moL} \mathrm{m}{ }^{-2} \mathrm{~s}^{-1}$ PAR with a $12 \mathrm{~h} \mathrm{light} / 12 \mathrm{~h}$ dark cycle. Individual plants with their root balls wrapped in aluminum foil were each placed in a single container. Air purified with a zero-air generator entered the container at a flow rate of $1.2 \mathrm{~L} \mathrm{~min}^{-1}$ and was actively pumped out of the container at a rate of $1.0 \mathrm{~L} \mathrm{~min}^{-1}$. Excess air was vented through a 2-mm opening at the top of the container. Air moisture was regulated by passing half of the incoming air through a washing flask containing distilled water. Volatiles were collected on $5 \mathrm{mg}$ activated charcoal traps (CLSA-Filter, Daumazan Sur Arize, France) placed directly in the outlet of the glass container.

For qualitative and quantitative analysis of floral volatiles, volatiles were sampled for $2 \mathrm{~h}$ with a closed-loop stripping system (Donath and Boland 1995; Tholl et al. 2005). Six to nine flowers were detached from individual plants and transferred to $10-\mathrm{mL}$ glass beakers filled with tap water and placed in a $1.5-\mathrm{L}$ glass desiccator in a plant climate chamber. Chamber conditions were as described for the other volatile collection system. Air circulated continuously with a flow rate of approximately $1 \mathrm{~L} \mathrm{~min}{ }^{-1}$, passing over 30-mg Super-Q (Alltech, Deerfield, IL, USA) traps.
Super-Q adsorbent was preferred over charcoal for floral volatile collection to prevent poor recovery of benzenoid compounds (Raguso and Pellmyr 1998). The recovery of benzaldehyde and phenylacetaldehyde on Super-Q adsorbent was analyzed by placing a $10 \mu \mathrm{L}$ mixture of both compounds (each $1 \mu \mathrm{g}$ dissolved in dichlormethane) at the bottom of a closed desiccator. Volatiles were collected for $2 \mathrm{~h}$ as described. Recovery of phenylacetaldehyde was $60 \%$ while benzaldehyde was recovered at $100 \%$.

Headspace solid-phase micro extraction (SPME) (Zhang and Pawliszyn 1993) was used for the analysis of volatiles from flower organs and enzyme products of terpene synthase assays. The fiber coating was PDMS-100 (100 $\mu \mathrm{m}$ polydimethylsiloxane) (Supelco, Bellefonte, PA, USA). Floral organs or enzyme solutions were placed in a 1-mL glass vial closed with a septum cap. The septum was punctured and the SPME needle inserted into the headspace of the sample. Samples were incubated at $25^{\circ} \mathrm{C}$ for floral volatile analysis and $30^{\circ} \mathrm{C}$ for enzyme assays and the fiber was exposed for $30 \mathrm{~min}$. Afterwards, the fiber was inserted into the injector of a gas chromatography-mass spectrometry (GC-MS) system for thermal desorption of the volatiles.

Volatiles collected on charcoal and Super-Q traps in the dynamic and closed-loop stripping systems were eluted with 50 and $100 \mu \mathrm{L} \mathrm{CH}_{2} \mathrm{Cl}_{2}$, respectively, containing 1-bromodecane or nonylacetate at a concentration of $2 \mathrm{ng} \mu \mathrm{L}^{-1}$ as a standard. One microliter of the eluate was analyzed with a Hewlett-Packard 6890 gas chromatograph coupled to a Hewlett-Packard 5973 quadrupole mass selective detector. Separation was performed on a (5\%-phenyl)methylpolysiloxane column (J\&W Scientific, Folstom, CA, USA) of $30 \times 0.25 \mathrm{~mm}$ i.d. $\times 0.25 \mu \mathrm{m}$ thickness. Helium was the carrier gas (flow rate of $2 \mathrm{~mL} \mathrm{~min}^{-1}$ ). A splitless injection was used, and a temperature program starting at $45^{\circ} \mathrm{C}$ with a ramp of $5^{\circ} \mathrm{C} \mathrm{min}^{-1}$ to $180^{\circ} \mathrm{C}$ followed by a ramp of $60^{\circ} \mathrm{C} \mathrm{min}^{-1}$ to $300^{\circ} \mathrm{C}$ was applied. Mass spectrometry was performed with a transfer line temperature of $230^{\circ} \mathrm{C}$, ion source temperature of $230^{\circ} \mathrm{C}$, quadrupole temperature of $150^{\circ} \mathrm{C}$, ionization potential of $70 \mathrm{eV}$, and scan range of 35 to 350 atomic mass units. Compounds were identified using the mass spectral libraries NIST98 (Agilent Technologies, Palo Alto, CA, USA) and Wiley275 and confirmed with authentic standards where available. For quantification, total ion peaks of each compound were integrated and the amount of compound was calculated based on external calibration curves generated with authentic standards. Emission rates for phenylacetaldehyde were corrected according to $60 \%$ recovery on Super-Q traps.

\section{Proton-transfer-reaction mass spectrometry}

Proton-transfer-reaction mass spectrometry (PTR-MS) allows an online detection of volatiles with a temporal 
resolution of less than $1 \mathrm{~min}$. A low fragmentation of analytes is achieved due to the low energy input used for protonation. Details of the analytical technique are described elsewhere (Lindinger et al. 1998; de Gouw et al. 2003). A mobile PTR-MS instrument (Ionicon Analytik, Innsbruck, Austria) was used for continuous measurement of volatiles emitted from intact plants of A. lyrata ssp. petraea. A single flowering plant (A) and a non-flowering plant (B) were each placed in a desiccator of 2-L volume. Desiccators were supplied with scrubbed (free of volatile organic carbons and ozone) and humidified air ( $\sim 50 \%$ relative humidity) at a flow rate of $1 \mathrm{~L} \mathrm{~min}^{-1}$. Light was provided by two sodium lamps (manually operated) and the temperature range was between $23^{\circ} \mathrm{C}$ (dark) and $28^{\circ} \mathrm{C}$ (light). Volatile emissions from $A$. lyrata flowers and leaves were measured continuously for $48 \mathrm{~h}$ ( 2 dark and light phases; $13 \mathrm{~h}$ light/ $11 \mathrm{~h}$ dark cycle) by alternating the air flow every $20 \mathrm{~min}$ between A and B with the help of an automatically controlled valve switch. The PTR-MS was run at a ratio of applied electric field to buffer gas number density $(\mathrm{E} / \mathrm{N})$ of $120 \mathrm{Td}$ and compounds of interest were quantified by monitoring characteristic protonated masses in selected ion mode for benzaldehyde (m107), phenylacetaldehyde (m121), monoterpenes (m81 and m137), methylsalicylate (m153), and sesquiterpenes (m149 and m205). PTR-MS data were corrected for mass discrimination and for the background signal measured in each empty desiccator. Total sesquiterpenes were calculated from the contribution of measured fragments relative to the total fragment masses.

Transcript analysis of the AtTPS21-homologous gene (AlCarS) in individuals of the Neutras A. lyrata ssp. petraea population and isolation of AlCarS cDNA

Total RNA was extracted from $1 \mathrm{~g}$ of leaves after P. xylostella feeding by using the TRIzol Reagent (Invitrogen, Carlsbad, CA, USA) as previously described (Herde et al. 2008). Four micrograms of total RNA were reverse transcribed into cDNA in a $20-\mu \mathrm{L}$ reaction with $0.5 \mu \mathrm{g}$ poly(dT) primer, $0.2 \mathrm{mM}$ of each dNTP, and 200 units of SuperScript III RNase $\mathrm{H}^{-}$Reverse Transcriptase (Invitrogen). Two primers, 5' -gcgatgacattcgtgagaatg- $3^{\prime}$ and 5'-ccttcaagagcttggggttg-3', specific for AtTPS 21 were used to amplify a 388-bp cDNA fragment with high similarity to AtTPS21. Next, a full-length cDNA of the putative A. lyrata (E)- $\beta$-caryophyllene synthase (AlCarS) was amplified by RT-PCR from RNA of the $(E)$ - $\beta$-caryophyllene-emitting plant line NT20 with the primers $5^{\prime}$-atggggag tgaagtcaaccgtc- $3^{\prime}$ and $5^{\prime}$-tcaaatgggtatagtttcaatgagcaa-3', which correspond to the start and the end of the coding region of AtTPS21. The similarity of the PCR products to AtTPS21 was confirmed by sequencing.
Genomic PCR analysis of AlCarS intergenic regions

Genomic DNA was isolated from leaves of A. lyrata ssp. petraea (NT20) according to Konieczny and Ausubel (1993). PCRs were performed with the AtTPS21/AlCarSspecific forward $\left(5^{\prime}\right.$-gcgatgacattcgtgagaatg- $\left.3^{\prime}\right)$ and reverse $\left(5^{\prime}\right.$-ccttcaagagcttggggttg- $\left.3^{\prime}\right)$ primers and the following primers positioned in the intergenic region upstream of AtTPS21 (igF1: 5' -cgcctaaaaatcttgtactc-3', igF2: 5' -tggttat actgttagtgag- $3^{\prime}$, igF3: $5^{\prime}$-ttttatacttgatgtaatcc-3', igF4: $5^{\prime}$-ag ctaataaatatctgaaatatc-3', igF5: $5^{\prime}$-tggacacacatatttcaag- $3^{\prime}$ ) and downstream of AtTPS21 at the $5^{\prime}$-end (R1: $5^{\prime}$-gcctctgg atgtcttcttgg- $3^{\prime}$ ) and $3^{\prime}$-end (R2: $5^{\prime}$-gatgaggatactaggacttgg- $3^{\prime}$ ) of gene At5g23970 and at the $3^{\prime}$-end of gene At5g23980 (on opposite strand, R3: 5' -cgttggggtgatggtttgcgg-3'). All possible combinations of forward and reverse primers were applied. PCRs were performed at different annealing temperatures with $50 \mathrm{ng}$ of DNA template, $0.4 \mu \mathrm{M}$ of each primer, $0.2 \mathrm{mM}$ of each dNTP, $1.5 \mathrm{mM}$ or $3 \mathrm{mM} \mathrm{MgCl}_{2}$, and $1 \mathrm{U}$ of Taq polymerase (Platinum Taq, Invitrogen).

Expression of AlCarS in E. coli and terpene synthase enzyme assay

For heterologous expression in E. coli BL21, the full-length coding region of the AlCarS cDNA was cloned in the expression vector pCR-T7/CT-TOPO (Invitrogen). Proteins were extracted from crude bacterial extract after $24 \mathrm{~h}$ cultivation at $18^{\circ} \mathrm{C}$ upon induction with $1 \mathrm{mM}$ IPTG using an extraction procedure described previously (Chen et al. 2003; Tholl et al. 2005). Terpene synthase enzyme assays with crude protein extracts and identification of TPS products by SPME and GC-MS analysis were performed under standard conditions as described (Chen et al. 2003; Tholl et al. 2005).

\section{Results}

Composition of Arabidopsis lyrata ssp. petraea floral odor

We collected floral scent from plants of an A. lyrata ssp. petraea population located in Southern Germany (Plech). Individual plants were derived from controlled crosses of sire and dam plants, which had been grown from seeds originally collected in the field (Clauss et al. 2006). Benzaldehyde and phenylacetaldehyde were the predominant volatile constituents (Fig. 1a, b). In addition, acetophenone was detected sporadically in small amounts (Fig. 1b).

We determined the qualitative and quantitative variation in floral volatile profiles among F1 plants of the Plech population (Fig. 1c). No major qualitative differences among volatile profiles were observed, and phenylacetaldehyde 


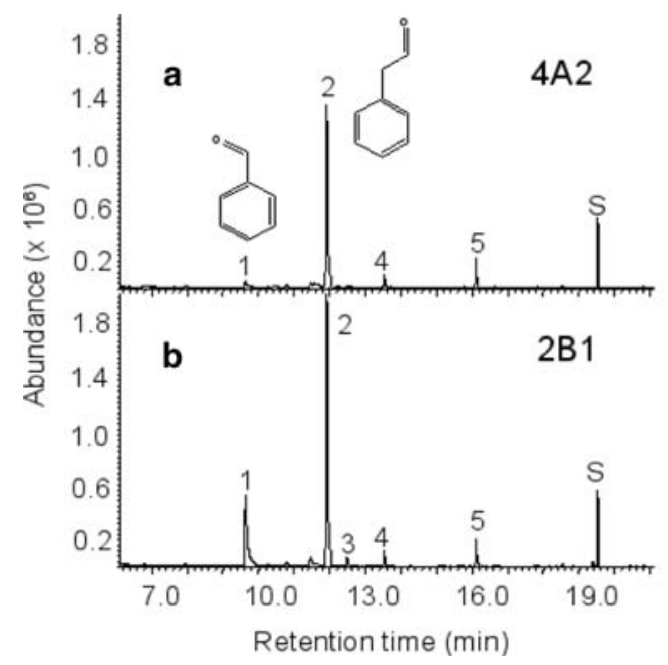

Fig. 1 Volatile emission from flowers of A. lyrata ssp. petraea. GC-MS chromatograms of volatiles collected from flowers of two individual plants 4A2 (a) and 2B1 (b) of a German A. lyrata ssp. petraea population (Plech). Volatiles were collected from six (a) or eight (b) detached flowers for $2 \mathrm{~h}$ (between $11 \mathrm{a} . \mathrm{m}$. and 1 p.m.) in a closed-loop stripping system as described under "Materials and methods". 1 benzaldehyde, 2 phenylacetaldehyde, 3 acetophenone, 4 nonanal, 5 decanal, $S$ internal

represented the predominant floral volatile compound in all plants. However, the absolute and relative emission rates of benzaldehyde and phenylacetaldehyde varied among individuals. Benzaldehyde was emitted at rates two- to ten-fold lower than those of phenylacetaldehyde. Differences in the emission between the highest and the lowest emitters of each compound were approximately twofold (Fig. 1c). Total emission rates (both compounds) varied between 51 and $115 \mathrm{ng}$ per $\mathrm{h}$ and flower.

To confirm the emission of benzenoids from floral organs, volatiles were trapped by SPME from detached flowers, flower petals and flowers from which petals had been removed (Fig. 2a-c). Benzenoid volatiles were detected exclusively from petal tissue (Fig. 2b) indicating that formation of these compounds in A. lyrata ssp. petraea flowers is organ specific.

\section{Rhythm of A. lyrata ssp. petraea floral scent emission}

Since it has been observed that A. lyrata ssp. petraea flowers are pollinated by day-active solitary bees and flies (Clauss and Koch 2006), we assumed that volatile emission from these flowers might occur during daylight. To analyze diurnal changes of A. lyrata ssp. petraea floral scent, volatile emissions were monitored online over $48 \mathrm{~h}$ from an intact flowering plant in comparison to a non-flowering plant using PTR-MS. Measurements showed light-dependent emissions of volatiles with protonated masses of 107 and 121 , which are indicative of benzaldehyde and phenylacetaldehyde, respectively. Signal intensities for these

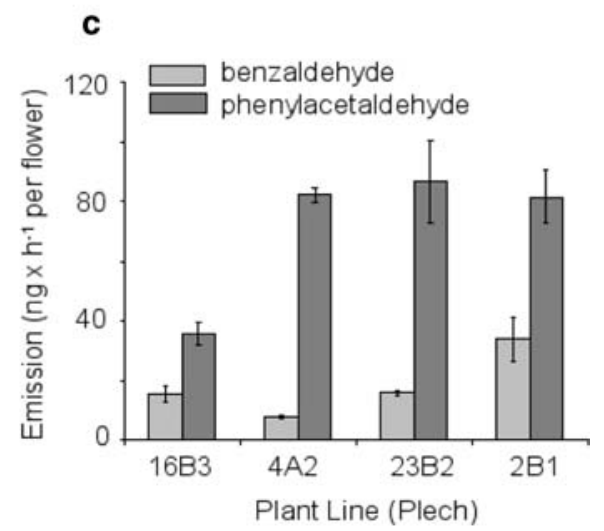

standard 1-bromodecane. Nonanal and decanal are background volatile contaminants. The chemical structures of benzaldehyde and phenylacetaldehyde are shown. $\mathbf{c}$ Emission rates of benzaldehyde and phenylacetaldehyde from different individuals of the Plech population. Calculations are based on volatile collections from three separate sets of flowers per plant

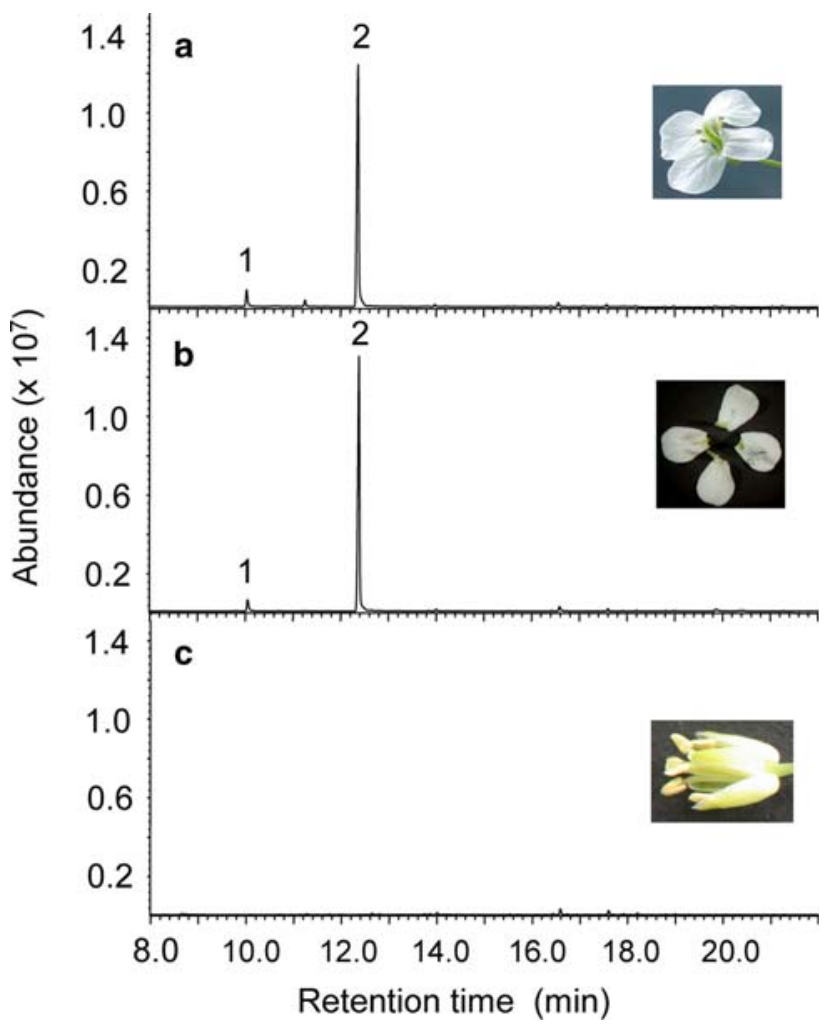

Fig. 2 Organ-specific emission of floral volatiles. GC-MS chromatograms of volatiles emitted from a single intact flower (a), the detached petals of a single flower (b), and a single flower from which petals were removed (c). Volatiles were trapped in 1-ml glass vials by solid-phase microextraction (SPME) as described under "Materials and methods". Flowers were from a Plech plant with an "4A2"-type volatile profile (see Fig. 1a, c) 
masses were not distinguishable from background when analyzed from the vegetative plant. Emission of phenylacetaldehyde from the flowering plant was approximately six- to ten-fold higher during the light period than in the dark phase (Fig. 3). The emission peaked approximately $1 \mathrm{~h}$ after the onset of light and began to decline approximately $4-5 \mathrm{~h}$ later. At the beginning of darkness, emission levels were already low and remained low until 2-3 $\mathrm{h}$ prior to the next light period. A similar pattern of emission was observed for benzaldehyde. In addition, a signal for $\mathrm{m} 205$ with an approximately 100-fold lower intensity than that of phenylacetaldehyde was detected which represents the sesquiterpene cyclosativene. This compound is emitted in low amounts primarily from foliage as determined by GC-MS analysis of volatiles from vegetative tissue (data not shown). The cyclosativene signal followed a less pronounced light-dependent emission pattern than that of the aromatic aldehydes with a 1.5-fold difference of emission between light and dark phases.

Emission of volatiles from A. lyrata ssp. petraea leaves upon feeding by Plutella xylostella

Given the obvious difference in floral volatile profiles between $A$. lyrata and A. thaliana species, we investigated whether there were also differences in volatiles emitted from leaves after insect feeding. To this end, we conducted a qualitative analysis of volatile blends from ten individual plants of an A. lyrata ssp. petraea population from Southern Germany (Neutras, Fränkische Schweiz) in response to feeding by larvae of the specialist-feeding lepidopteran

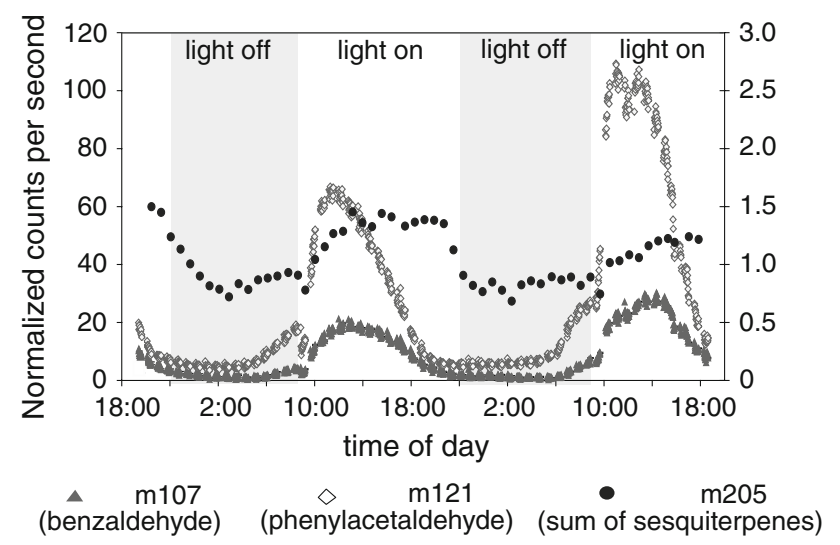

Fig. 3 Diurnal changes of floral and vegetative volatile emissions from A. lyrata ssp. petraea. High resolution volatile measurement recorded by PTR-MS for a single intact flowering plant (Plech). Volatile emission was monitored continuously over a time course of $48 \mathrm{~h}$ under a $13 \mathrm{~h} \mathrm{light} / 11 \mathrm{~h}$ dark rhythm as described in "Materials and methods". The protonated masses of benzaldehyde (107), phenylacetaldehyde (121), and sesquiterpene olefins including cyclosativene (205) are recorded. The scale for mass 205 is shown on the right
Plutella xylostella (Fig. 4). Rosette leaves of four of the analyzed plants emitted the sesquiterpene $(E)$ - $\beta$-caryophyllene accompanied by minor amounts of $\alpha$-humulene. Other induced volatiles were the homoterpenes DMNT and TMTT, the sesquiterpene nerolidol and methyl salicylate. Emission of these compounds varied between individual plants with TMTT found in seven, DMNT in six, nerolidol in five, and methyl salicylate in four individuals. Volatile profiles of two plants emitting $(E)-\beta$-caryophyllene are shown in Fig. 4a, b.

Enzyme activity of an herbivore-induced A. lyrata ssp. petraea terpene synthase with high similarity

to A. thaliana TPS 21 (At5g23960)

In contrast to A. lyrata, A. thaliana emits the sesquiterpene (E)- $\beta$-caryophyllene exclusively from flowers and not from leaf tissue (Chen et al. 2003). To investigate the molecular basis of this difference, we searched for a $(E)$ - $\beta$-caryophyllene synthase in $A$. lyrata ssp. petraea leaves. Using primers specific for the florally expressed $A$. thaliana $(E)-\beta$ caryophyllene synthase (AtTPS21, At5g23960, GenBank accession number AF497491) (Aubourg et al. 2002), we detected a 388-bp cDNA fragment in rosette leaves of $(E)$ $\beta$-caryophyllene-emitting $A$. lyrata plants $20 \mathrm{~h}$ after the beginning of $P$. xylostella feeding (Fig. 5). This PCR product showed high sequence identity to A. thaliana $(E)-\beta$ caryophyllene synthase and was absent in plant lines that did not emit (E)- $\beta$-caryophyllene (Fig. 5). A full-length cDNA of the AtTPS21-similar gene designated AlCarS (GenBank accession number FJ719785) was isolated from line NT20 by RT-PCR. The coding sequence of AlCarS consists of 1,638 nucleotides corresponding to 545 amino acids. AtTPS21 and AlCarS share 93 and 91\% identity at the nucleotide sequence and amino acid sequence level, respectively, and differ in length by two amino acids (Fig. 6).

To determine the biochemical function of the AlCarS protein, the corresponding full-length cDNA was expressed heterologously in E. coli. A terpene synthase assay of the recombinant protein yielded $(E)$ - $\beta$-caryophyllene as the major enzymatic product with farnesyl diphosphate (FPP) as the substrate (Fig. 7). $\alpha$-Humulene and $\alpha$-copaene were detected as additional products in smaller amounts.

To examine intergenic orthologous regions of AlCarS and $A$. thaliana AtTPS21, PCRs were conducted with genomic DNA extracted from line NT20 using combinations of primers specific for AlCarS and primers positioned in the intergenic region and neighboring genes up- and downstream of AtTPS21, respectively. No PCR products were amplified with different primer combinations suggesting differential genomic positions of AlCarS and AtTPS21 in the two Arabidopsis species. 


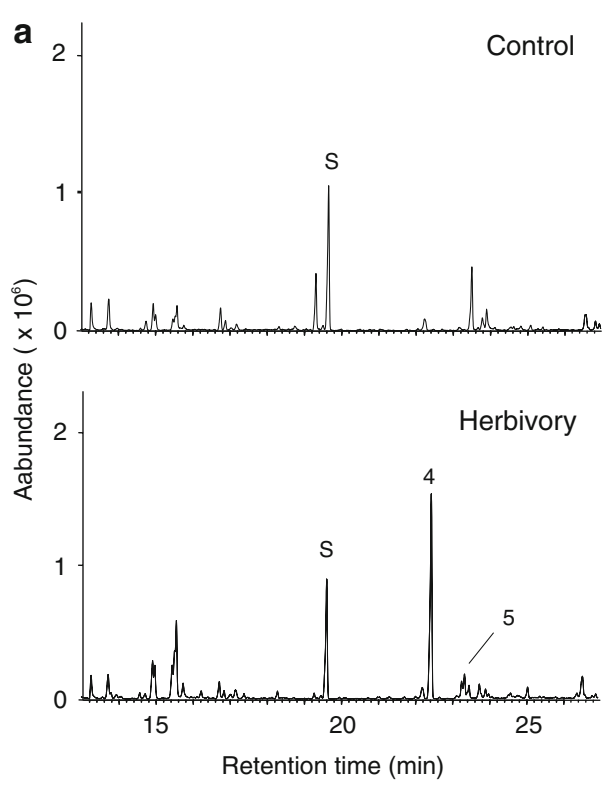

Fig. 4 Insect-induced volatile emissions from leaves of A. lyrata ssp. petraea. 20-30 P. xylostella larvae were placed on rosette leaves of individual non-flowering plants (population Neutras, Germany) and removed $24 \mathrm{~h}$ later. Volatiles were collected from control plants (upper panel) and insect-damaged plants (lower panel) of two selected lines, NT20 (a) and NT51 (b) as described under "Materials and methods".

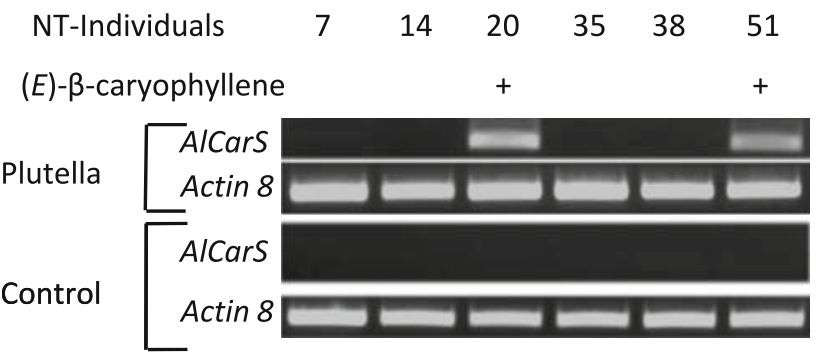

Fig. 5 Herbivore-induced transcription of an A. thaliana (E)- $\beta$-caryophyllene synthase gene homologue in A. lyrata ssp. petraea rosette leaves. Semi-quantitative RT-PCR analysis of transcript levels of an $A$. lyrata ssp. petraea gene (AlCarS) with high sequence similarity to the A. thaliana (E)- $\beta$-caryophyllene synthase (At5g23960/AtTPS21). Induced transcripts were observed only in rosette leaves of plants that emitted (E)- $\beta$-caryophyllene after $20 \mathrm{~h}$ of feeding by $P$. xylostella. Control reactions were performed with RNA from undamaged clonal plants of the same line. Reactions with primers for actin- 8 were performed to judge equality of the cDNA template concentration

\section{Discussion}

Floral scent of A. lyrata ssp. petraea is different from that of $A$. thaliana and is dominated by benzenoid compounds

Within the Brassicaceae family, A. lyrata and A. thaliana are emerging as a model system for comparative analyses in plant evolution and ecology. Given the differences in the

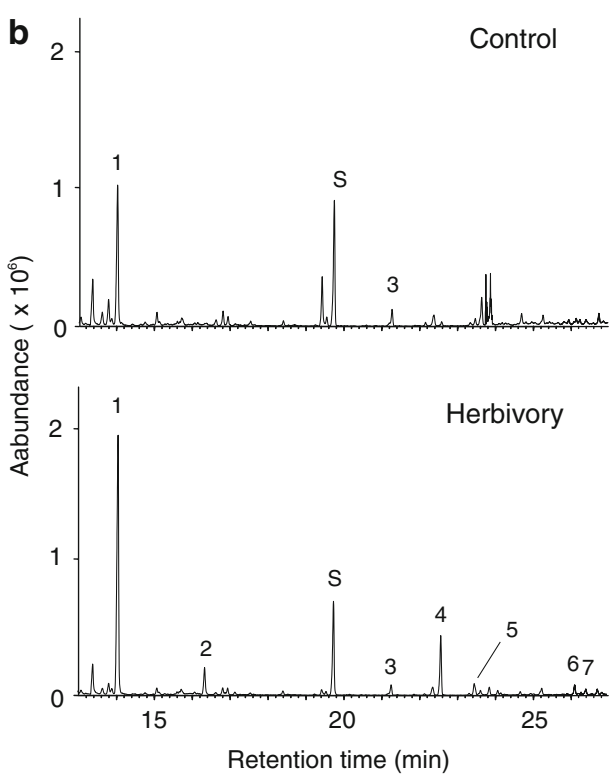

GC-MS chromatograms are shown only for volatiles collected on day 2 since emission rates were highest on this day. 1 DMNT, 2 methyl salicylate, $3(+)$-cyclosativene, $4(E)$ - $\beta$-caryophyllene, $5 \alpha$-humulene, 6 nerolidol, 7 TMTT, $S$ nonyl acetate standard. Unlabeled peaks represent background volatiles or compounds whose identity could not be precisely determined by library comparison

ecological niche of the two species, it may be expected that their pollinator and herbivore communities are quite distinct.

We have analyzed the floral volatile profiles of A. lyrata ssp. petraea plants from a population in central Europe. The floral scent of these plants is detectable by the human nose and contains benzenoids as the predominant compounds. The most abundant fragrance constituent is phenylacetaldehyde which was also reported as a major floral volatile in the North American subspecies A. lyrata ssp. lyrata indicating that emission of this compound is a conserved trait of the two A. lyrata subspecies. Another volatile found in both subspecies is benzaldehyde, but other compounds emitted from A. lyrata ssp. lyrata flowers such as $\mathrm{C}_{6}$ alcohols, phenylethanols, and the monoterpene myrcene (Peer and Murphy 2003) are absent in A. lyrata ssp. petraea floral volatile blends as described.

Benzenoids and phenylpropanoids are common constituents of floral scent in many plant families including the Brassicaceae (Blight et al. 1997; Omura et al. 1999; Jönsson et al. 2005; Knudsen et al. 2006). Both classes of compounds elicit olfactory responses and contribute to the attraction of pollinating butterflies (Omura et al. 1999), bees (Blight et al. 1997) and moths (Raguso and Light 1998; Meagher 2002; Hoballah et al. 2005). Therefore, it is likely that these volatiles also serve as olfactory cues in the attraction of the solitary bees, flies, and butterflies that have been observed as pollinators in European and North American 
Fig. 6 Protein sequence comparison of AlCarS with A. thaliana AtTPS21. Amino acid sequences of AlCarS and AtTPS 21 are $91 \%$ identical. Identical amino acids are shaded in black. Dashes indicate gaps inserted for optimal alignment. The DDXXD motif that is highly conserved among class I terpene synthases and obligatory for catalytic activity is underlined

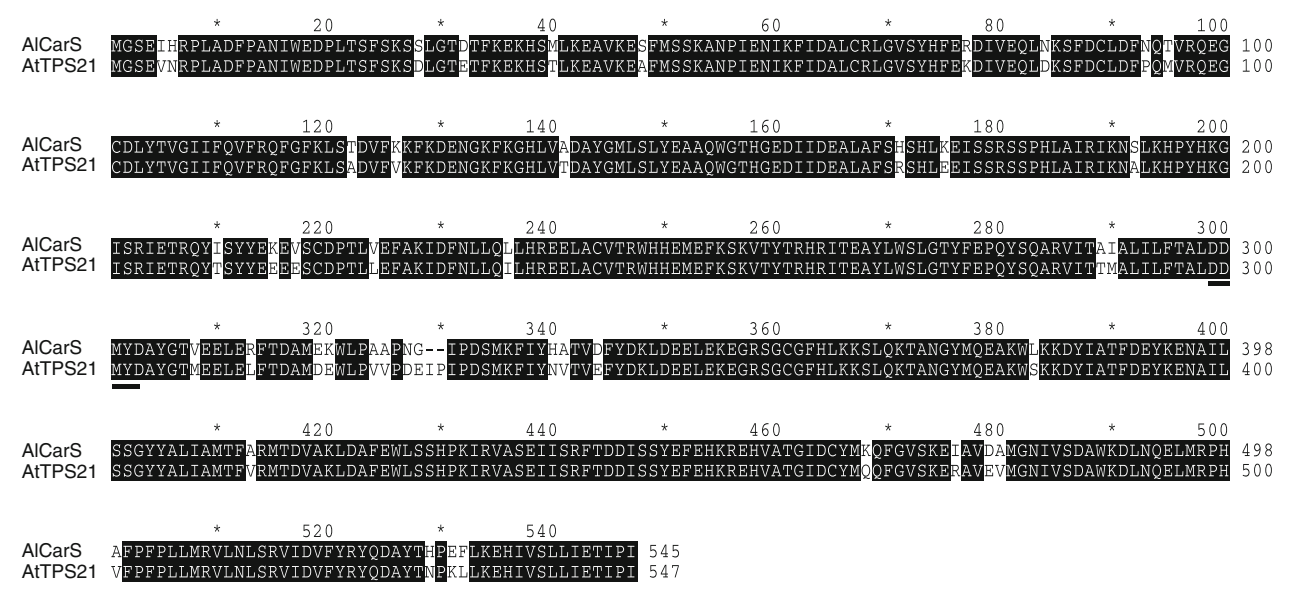

A. lyrata populations (Clauss and Koch 2006; Peer and Murphy 2003).

In contrast to A. lyrata flowers, the volatile compounds of A. thaliana flowers are primarily monoterpenes and a complex mix of sesquiterpenes with $(E)$ - $\beta$-caryophyllene as the major component (Chen et al. 2003; Tholl et al. 2005). However, emissions of $(E)-\beta$-caryophyllene from single inflorescences (containing 3-4 open flowers) of the highestemitting A. thaliana accessions investigated are approximately 260 -fold lower than the highest phenylacetaldehyde emissions from the same number of flowers of A. lyrata ssp. petraea (Plech population). Because of these low emission rates, A. thaliana floral volatiles may be effective only in the short-range attraction of pollinating insects that contribute to the small percentage of outcrossing observed in natural populations (Snape and Lawrence 1971; Abbott and Gomes 1989; Hoffmann et al. 2003). A putative defense function of sesquiterpene volatiles in A. thaliana flowers is

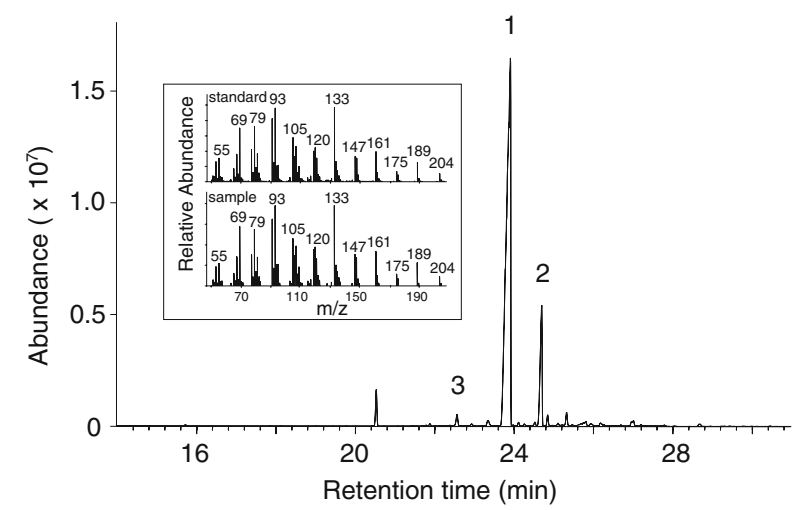

Fig. 7 Formation of (E)- $\beta$-caryophyllene by recombinant AlCarS enzyme. AlCarS was expressed in $E$. coli, extracted, and incubated with the substrate all-trans-FPP. The resulting terpene products were analyzed by GC-MS. The major product was identified as $(E)$ - $\beta$-caryophyllene (1). Other products were $\alpha$-humulene (2) and $\alpha$-copaene (3). The mass spectrum of the $(E)-\beta$-caryophyllene product is shown in comparison to that of an authentic $(E)$ - $\beta$-caryophyllene standard (inset) suggested by their specific formation in the stigma and floral nectaries (Tholl et al. 2005), organs that are possible sites of microbial invasion (Thornburg et al. 2003). In contrast, A. lyrata ssp. petraea flowers (Plech population) emit benzaldehyde and phenylacetaldehyde only from petal tissue similar to several insect-pollinated plant species such as Antirrhinum majus, Petunia $\times$ hybrida and Clarkia breweri (Dudareva et al. 1998, 2000; Verdonk et al. 2003). Interestingly, we have detected small amounts of benzaldehyde $\left(\leq 0.3 \mathrm{ng}\right.$ inflorescence $\left.{ }^{-1} \mathrm{~h}^{-1}\right)$ in flowers of several A. thaliana ecotypes (unpublished results) but its site of emission in the A. thaliana flower is not known. Overall, our observations are consistent with the notion that the formation of benzenoid volatiles in flower petals was under positive selection in the evolution of A. lyrata ssp. petraea as a strictly outcrossing species to aid in efficient attraction of pollinators. In comparison, during the evolution of A. thaliana as a largely selfing species the selection pressure for this trait may not have been high or negative resulting in the production of only trace amounts of aromatic pollinator attractants.

Interspecific differences in the qualitative emission of benzenoid and terpenoid compounds have also been described in the genus Nicotiana (Raguso et al. 2003). For example, $(E)$ - $\beta$-caryophyllene and 1,8-cineole are abundant in flowers of several Nicotiana species such as $N$. suaveolens but absent in others. Similar to $(E)$ - $\beta$-caryophyllene produced in A. thaliana flowers, 1.8-cineole is in part released from the stigma and style (Roeder et al. 2007) suggesting similar putative defense functions.

Despite the previously reported high genetic diversity among individuals of Central European A. lyrata ssp. petraea populations (Clauss and Mitchell-Olds 2006), we found almost no qualitative differences in floral volatile composition within the analyzed population. Variation was apparent only at quantitative levels with an approx. twofold difference in emission rates of each aromatic compound and a two- to ten-fold difference in their emission relative to 
each other (Fig. 2c). The apparent population-specific conservation of the floral volatile composition supports the significance of these volatile blends in the sustainability of the analyzed population.

Previous inter-population studies of several A. lyrata ssp. petraea populations in Northern and Central Europe demonstrated distinct primary metabolite and glucosinolate phenotypes correlated with genetic differences between these populations (Davey et al. 2008). We have observed qualitative and quantitative variation of benzenoid and terpene floral volatiles between the Plech population and two populations from Austria and the Czech republic (unpublished results) that might reflect responses to distinct local pollinator or herbivore faunas or be the result of random genetic drift in isolated populations (Tollsten and Bergström 1993; Mant et al. 2005; Jhumur et al. 2008).

Emission of A. lyrata ssp. petraea floral volatiles follows a diurnal rhythm

The analysis of benzaldehyde and phenylacetaldehyde emissions from A. lyrata ssp. petraea flowers revealed a diurnal pattern for both compounds with highest emission $2 \mathrm{~h}$ after the onset of light. The diurnal volatile release coincides with the observed visits of $A$. lyrata flowers by solitary bees and syrphid flies and support the role of floral volatiles in the attraction of such pollinators (Clauss and Koch 2006). Our findings are in agreement with previous studies demonstrating that flowers pollinated by bees or other day-active insects tend to emit volatiles during daylight with highest emission rates at midday (Matile and Altenburger 1988). In contrast, hawkmoth-pollinated plants such as Petunia and Nicotiana species show maximum output of benzenoid and terpenoid (Nicotiana) floral volatiles during the night (Loughrin et al. 1991; Kolosova et al. 2001; Raguso et al. 2003; Hoballah et al. 2005; Roeder et al. 2007) of which benzenoids in particular have been shown to elicit pollinator olfactory responses (Hoballah et al. 2005). However, not all scent components with a rhythmic emission pattern may be involved in pollinator attraction as shown, for example, by the absence of hawkmoth antennal perception to two nocturnally emitted benzenoid compounds of Petunia axillaris flowers (Hoballah et al. 2005). Moreover, not all floral volatiles are necessarily released in a rhythmic manner (Hoballah et al. 2005). It is possible that other factors such as anti-herbivore defense, pathogen resistance or predator avoidance have contributed to the evolution of floral volatile formation and emission patterns including those of A. lyrata ssp. petraea.

As demonstrated by PTR-MS analysis, A. lyrata ssp. petraea flowers release benzaldehyde and phenylacetaldehyde in a synchronous manner (Fig. 3) suggesting coregulation of their biosynthetic pathways. Whether the formation of both compounds is controlled by an endogenous circadian clock (Kolosova et al. 2001) remains to be determined by analyzing volatile emission and the expression of biosynthetic enzymes under continuous light and dark regimes.

Arabidopsis lyrata ssp. petraea emits terpenoids from foliage upon insect feeding employing an (E)- $\beta$-caryophyllene synthase with high similarity to a flower-specific A. thaliana $(E)$ - $\beta$-caryophyllene synthase

Feeding of the crucifer specialist $P$. xylostella (diamond back moth) on leaves of $A$. lyrata ssp. petraea from Southern Germany (Neutras) induces variable emission of the sesquiterpenes $(E)$ - $\beta$-caryophyllene and nerolidol, the homoterpenes 4,8-dimethyl-1,3,7-nonatriene (DMNT) and 4,8,12-trimethyltrideca-1,3,7,11-tetraene (TMTT), and methyl salicylate (MeSA) (Fig. 4a, b). The released volatiles may aid in the attraction of insect parasitoids as was demonstrated for the volatile blend emitted from A. thaliana leaves in response to feeding by Pieris rapae (Van Poecke et al. 2001). TMTT is also emitted from A. thaliana leaves upon Pieris rapae and P. xylostella feeding (Van Poecke et al. 2001; Herde et al. 2008). However, nerolidol and its degradation product DMNT are absent in A. thaliana leaf volatile blends induced by both insects (Van Poecke et al. 2001; Herde et al. 2008). This difference might result from the differential expression of a nerolidol synthase catalyzing the first committed step in DMNT formation since no induced transcript of such terpene synthase has been found in A. thaliana leaves upon feeding damage (Herde et al. 2008).

Another difference between the volatile blends of A. lyrata and A. thaliana is apparent in the emission of (E)- $\beta$-caryophyllene. (E)- $\beta$-Caryophyllene is a common floral volatile (Knudsen et al. 2006), and it is involved in the attraction of insect-parasitizing wasps and nematodes when released from leaves and roots, respectively (Rasmann et al. 2005; Köllner et al. 2008).

In A. lyrata, (E)- $\beta$-caryophyllene is produced by the foliage upon herbivory but is absent from flowers while most $A$. thaliana ecotypes emit (E)- $\beta$-caryophyllene as a floral volatile (Tholl et al. 2005) but not from rosette leaves after attack by $P$. rapae or P. xylostella (Van Poecke et al. 2001; Herde et al. 2008). We could show that the biosynthesis of (E)- $\beta$-caryophyllene in these two Arabidopsis species is catalyzed by genes with high sequence identity. Insectinduced emission of $(E)$ - $\beta$-caryophyllene varies among individuals of the investigated A. lyrata ssp. petraea population and correlates with the presence or absence of the caryophyllene synthase (AlCarS) transcript (Fig. 5). A similar regulation of $(E)$ - $\beta$-caryophyllene emission at the gene transcript level has been reported from European and North 
American maize varieties that differ in their ability to produce this volatile in leaves and roots upon insect attack (Köllner et al. 2008). Future studies should determine to which degree changes in cis- and/or trans-regulatory elements of the two caryophyllene synthase genes in A. thaliana and A. lyrata led to their organ-specific and inter-/intra-population differences in expression.

It can be assumed that AlCarS and AtTPS21 represent orthologous genes. However, orthologous intergenic regions could not be confirmed by genomic PCR using gene-specific primers and several primers positioned in the intergenic regions and neighboring genes of AtTPS21. It is possible that the A. lyrata ssp. petraea genome in contrast to $A$. thaliana carries more than a single caryophyllene synthase gene although only a single AlCarS cDNA was amplified by RT-PCR. Alternatively, different chromosomal positions of the genes in the two species could be the result of chromosome rearrangements in the A. thaliana genome (Blanc et al. 2000). Further analysis of the genome position of AlCarS supported by information from the A. lyrata genome sequencing project will aid in assessing AlCarS/ AtTPS21 gene evolution.

We assume that the ability to emit $(E)$ - $\beta$-caryophyllene as a floral volatile existed in the progenitor of both Arabidopsis species since this sesquiterpene was detected in flowers of the Arabidopsis-related species Boechera holboellii (Raguso and Roy 1998) and Boechera drummondii (personal observation) which diverged from the genus Arabidopsis more than 10 million years ago (Koch et al. $2001)$. Whether these species also produce $(E)$ - $\beta$-caryophyllene in response to insect feeding is not known. We propose a possible evolutionary scenario according to which the formation of $(E)$ - $\beta$-caryophyllene persisted in flowers of A. thaliana, but it gave way to the biosynthesis of benzenoids in A. lyrata flowers for more efficient pollinator attraction. In contrast, the insect-induced release of (E)- $\beta$-caryophyllene in leaves of A. lyrata may represent part of a more complex volatile response of this perennial to herbivores, while it did not evolve in A. thaliana because of its short life cycle and limited exposure of its foliage to herbivores and their natural enemies. The observed variation of the insect-induced $(E)$ - $\beta$-caryophyllene emission among individuals of the analyzed A. lyrata population might reflect the costs and benefits of volatile emission under fluctuating herbivore pressure. In the Plech population, Clauss et al. (2006) found significant genetic variation for amounts and ratios of glucosinolates and a positive correlation with insect resistance. For a greater understanding of the evolution of (E)- $\beta$-caryophyllene formation in Arabidopsis, further analyses of the biosynthesis of $(E)$ - $\beta$-caryophyllene in flowers and leaves of other A. lyrata populations and Arabidopsis-related species are required.
Acknowledgments We are thankful to Heiko Vogel (Max Planck Institute for Chemical Ecology, Jena, Germany) for providing Plutella xylostella larvae. We thank Katrin Luck, Isabel Putsche, and Bettina Raguschke for excellent laboratory assistance and Andreas Weber for maintaining the plant cultures (all MPI for Chemical Ecology, Jena, Germany). We are grateful to Michael Reichelt (MPI for Chemical Ecology) and Wolfgang Weisser for helpful comments during preparation of the manuscript. Financial support was received from the MaxPlanck-Gesellschaft (to C.A., M.C., D.T., J.G.), from Virginia Tech (to D.T.) and from VOCBAS and ISONET to A.S.

Open Access This article is distributed under the terms of the Creative Commons Attribution Noncommercial License which permits any noncommercial use, distribution, and reproduction in any medium, provided the original author(s) and source are credited.

\section{References}

Abbott RJ, Gomes MF (1989) Population genetic structure and outcrossing rate of Arabidopsis thaliana (L) Heynh. Heredity 62:411-418

Aubourg S, Lecharny A, Bohlmann J (2002) Genomic analysis of the terpenoid synthase (AtTPS) gene family of Arabidopsis thaliana. Mol Genet Genomics 267:730-745

Blanc G, Barakat A, Guyot R, Cooke R, Delseny I (2000) Extensive duplication and reshuffling in the Arabidopsis genome. Plant Cell 12:1093-1101

Blight MM, LeMetayer M, Delegue MHP, Pickett JA, Marion-Poll F, Wadhams LJ (1997) Identification of floral volatiles involved in recognition of oilseed rape flowers, Brassica napus by honeybees, Apis mellifera. J Chem Ecol 23:1715-1727

Chen F, Tholl D, D'Auria JC, Farooq A, Pichersky E, Gershenzon J (2003) Biosynthesis and emission of terpenoid volatiles from Arabidopsis flowers. Plant Cell 15:481-494

Clauss MJ, Koch MA (2006) Poorly known relatives of Arabidopsis thaliana. Trends Plant Sci 11:449-459

Clauss MJ, Mitchell-Olds T (2006) Population genetic structure of Arabidopsis lyrata in Europe. Mol Ecol 15:2753-2766

Clauss MJ, Dietel S, Schubert G, Mitchell-Olds T (2006) Glucosinolate and trichome defenses in a natural Arabidopsis lyrata population. J Chem Ecol 32:2351-2373

Davey MP, Burrell MM, Woodward FI, Quick WP (2008) Populationspecific metabolic phenotypes of Arabidopsis lyrata ssp petraea. New Phytol 177:380-388

de Gouw J, Warneke C, Karl T, Eerdekens G, van der Veen C, Fall R (2003) Sensitivity and specificity of atmospheric trace gas detection by proton-transfer-reaction mass spectrometry. Int J Mass Spectrom 223:365-382

Degen T, Dillmann C, Marion-Poll F, Turlings TCJ (2004) High genetic variability of herbivore-induced volatile emission within a broad range of maize inbred lines. Plant Physiol 135:1928-1938

Dicke M, van Poecke RMP, de Boer JG (2003) Inducible indirect defence of plants: from mechanisms to ecological functions. Basic Appl Ecol 4:27-42

Donath J, Boland W (1995) Biosynthesis of acyclic homoterpenesenzyme selectivity and absolute configuration of the nerolidol precursor. Phytochemistry 39:785-790

Dudareva N, Raguso RA, Wang JH, Ross EJ, Pichersky E (1998) Floral scent production in Clarkia breweri III-enzymatic synthesis and emission of benzenoid esters. Plant Physiol 116:599-604

Dudareva N, Murfitt LM, Mann CJ, Gorenstein N, Kolosova N, Kish CM, Bonham C, Wood K (2000) Developmental regulation of methyl benzoate biosynthesis and emission in snapdragon flowers. Plant Cell 12:949-961 
Fäldt J, Arimura G, Gershenzon J, Takabayashi J, Bohlmann J (2003) Functional identification of AtTPSO3 as $(E)$-beta-ocimene synthase: a monoterpene synthase catalyzing jasmonate- and woundinduced volatile formation in Arabidopsis thaliana. Planta 216:745-751

Gouinguene S, Degen T, Turlings TCJ (2001) Variability in herbivoreinduced odour emissions among maize cultivars and their wild ancestors (teosinte). Chemoecology 11:9-16

Herde M, Gärtner K, Köllner TG, Fode B, Boland W, Gershenzon J, Gatz C, Tholl D (2008) Identification and regulation of TPS04/ GES, an Arabidopsis geranyllinalool synthase catalyzing the first step in the formation of the insect-induced volatile C-16-homoterpene TMTT. Plant Cell 20:1152-1168

Hoballah ME, Stuurman J, Turlings TCJ, Guerin PM, Connetable S, Kuhlemeier C (2005) The composition and timing of flower odour emission by wild Petunia axillaris coincide with the antennal perception and nocturnal activity of the pollinator Manduca sexta. Planta 222:141-150

Hoffmann MH, Glass AS, Tomiuk J, Schmuths H, Fritsch RM, Bachmann K (2003) Analysis of molecular data of Arabidopsis thaliana (L.) Heynh. (Brassicaceae) with geographical information systems (GIS). Mol Ecol 12:1007-1019

Iijima Y, Davidovich-Rikanati R, Fridman E, Gang DR, Bar E, Lewinsohn E, Pichersky E (2004) The biochemical and molecular basis for the divergent patterns in the biosynthesis of terpenes and phenylpropenes in the peltate glands of three cultivars of basil. Plant Physiol 136:3724-3736

Jhumur US, Dötterl S, Jürgens A (2008) Floral odors of Silene otites: their variability and attractiveness to mosquitoes. J Chem Ecol 34:14-25

Jönsson M, Lindkvist A, Anderson P (2005) Behavioural responses in three ichneumonid pollen beetle parasitoids to volatiles emitted from different phenological stages of oilseed rape. Entomol Exp Appl 115:363-369

Kessler A, Baldwin IT (2001) Defensive function of herbivore-induced plant volatile emissions in nature. Science 291:2141-2144

Knudsen JT, Eriksson R, Gershenzon J, Stahl B (2006) Diversity and distribution of floral scent. Bot Rev 72:1-120

Koch MA, Haubold B, Mitchell-Olds T (2000) Comparative evolutionary analysis of chalcone synthase and alcohol dehydrogenase loci in Arabidopsis, Arabis, and related genera (Brassicaceae). Mol Biol Evol 17:1483-1498

Koch M, Haubold B, Mitchell-Olds T (2001) Molecular systematics of the Brassicaceae: evidence from coding plastidic $m a t K$ and nuclear Chs sequences. Am J Bot 88:534-544

Köllner TG, Held M, Lenk C, Hiltpold I, Turlings TCJ, Gershenzon J, Degenhardt J (2008) A maize (E)-beta-caryophyllene synthase implicated in indirect defense responses against herbivores is not expressed in most American maize varieties. Plant Cell 20:482-494

Kolosova N, Gorenstein N, Kish CM, Dudareva N (2001) Regulation of circadian methyl benzoate emission in diurnally and nocturnally emitting plants. Plant Cell 13:2333-2347

Konieczny A, Ausubel FM (1993) A procedure for mapping Arabidopsis mutations using codominant ecotype-specific PCR-based markers. Plant J 4:403-410

Lindinger W, Hansel A, Jordan A (1998) Online monitoring of volatile organic compounds at pptv levels by means of proton-transferreaction mass spectrometry (PTR-MS) - medical applications, food control and environmental research. Int J Mass Spectrom 173:191-241

Loughrin JH, Hamiltonkemp TR, Andersen RA, Hildebrand DF (1991) Circadian rhythm of volatile emission from flowers of Nicotiana sylvestris and $N$ suaveolens. Physiol Plant 83:492-496
Mant J, Peakall R, Schiestl FP (2005) Does selection on floral odor promote differentiation among populations and species of the sexually deceptive orchid genus Ophrys? Evolution 59:1449-1463

Matile P, Altenburger R (1988) Rhythms of fragrance emission in flowers. Planta 174:242-247

Meagher RL (2002) Trapping noctuid moths with synthetic floral volatile lures. Entomol Exp Appl 103:219-226

Nasrallah ME (2000) Arabidopsis species hybrids-emerging model systems for the analysis of species differences. J Plant Growth Regul 19:326-333

Omura H, Honda K, Hayashi N (1999) Chemical and chromatic bases for preferential visiting by the cabbage butterfly, Pieris rapae, to rape flowers. J Chem Ecol 25:1895-1906

Peer WA, Murphy AS (2003) Floral scent of Arabidopsis lyrata (Brassicaceae). Biochem Syst Ecol 31:1193-1195

Pichersky E, Gershenzon J (2002) The formation and function of plant volatiles: perfumes for pollinator attraction and defense. Curr Opin Plant Biol 5:237-243

Raguso RA, Light DM (1998) Electroantennogram responses of male Sphinx perelegans hawkmoths to floral and 'green-leaf volatiles'. Entomol Exp Appl 86:287-293

Raguso RA, Pellmyr O (1998) Dynamic headspace analysis of floral volatiles: a comparison of methods. Oikos 81:238-254

Raguso RA, Roy BA (1998) 'Floral' scent production by Puccinia rust fungi that mimic flowers. Mol Ecol 7:1127-1136

Raguso RA, Levin RA, Foose SE, Holmberg MW, Mcdade LA (2003) Fragrance chemistry, nocturnal rhythms and pollination "syndromes" in Nicotiana. Phytochemistry 63:265-284

Rasmann S, Köllner T, Degenhardt J, Hiltpold I, Toepfer S, Kuhlmann U, Gershenzon J, Turlings T (2005) Recruitment of entomopathogenic nematodes by insect-damaged maize roots. Nature 434:732-737

Ratzka A, Vogel H, Kliebenstein DJ, Mitchell-Olds T, Kroymann J (2002) Disarming the mustard oil bomb. Proc Natl Acad Sci USA 99:11223-11228

Roeder S, Hartmann AM, Effmert U, Piechulla B (2007) Regulation of simultaneous synthesis of floral scent terpenoids by the 1,8-cineole synthase of Nicotiana suaveolens. Plant Mol Biol 65:107-124

Snape JW, Lawrence MJ (1971) Breeding system of Arabidopsis thaliana. Heredity 27:299-301

Tholl D, Chen F, Petri J, Gershenzon J, Pichersky E (2005) Two sesquiterpene synthases are responsible for the complex mixture of sesquiterpenes emitted from Arabidopsis flowers. Plant J 42:757771

Thornburg RW, Carter C, Powell A, Mittler R, Rizhsky L, Horner HT (2003) A major function of the tobacco floral nectary is defense against microbial attack. Plant Syst Evol 238:211-218

Tollsten L, Bergström LG (1993) Fragrance chemotypes of Platanthera (Orchidaceae) - the result of adaptation to pollinating moths. Nord J Bot 13:607-613

Van Poecke RMP, Posthumus MA, Dicke M (2001) Herbivore-induced volatile production by Arabidopsis thaliana leads to attraction of the parasitoid Cotesia rubecula: chemical, behavioral, and gene-expression analysis. J Chem Ecol 27:1911-1928

Verdonk JC, de Vos CHR, Verhoeven HA, Haring MA, van Tunen AJ, Schuurink RC (2003) Regulation of floral scent production in petunia revealed by targeted metabolomics. Phytochemistry 62:997-1008

Zhang ZY, Pawliszyn J (1993) Headspace solid-phase microextraction. Anal Chem 65:1843-1852 